EUROPEAN ORGANIZATION FOR NUCLEAR RESEARCH
Laboratory for Particle Physics

Departmental Report

CERN/AT 2007-28 (MTM)

\title{
ERROR MODELING OF A FAST DIGITAL INTEGRATOR FOR MAGNETIC MEASUREMENTS AT CERN
}

\author{
P. Arpaia ${ }^{1}$, V. Inglese ${ }^{2,3}$, Gi. Spiezia ${ }^{2,3}$, S. Tiso ${ }^{3}$
}

\begin{abstract}
A statistical behavioral modeling approach for assessing dynamic metrological performance during the concept design of accurate digitizers is proposed. A surface-response approach based on statistical experiment design is exploited in order to avoid unrealistic hypothesis of linearity, optimize simulation effort, explore systematically operating conditions, and verify identification and validation uncertainty. An actual case study on the dynamic metrological characterization of a Fast Digital Integrator for highperformance magnetic measurements at European Organization for Nuclear Research (CERN) is presented.
\end{abstract}

1 University of Sannio, Department of Engineering, Benevento, Italy 2 University of Naples, Department of Engineering, Napoli, Italy

3 CERN, Accelerator Technology Department, Geneva, Switzerland

Presented at the Instrumentation and Measurement Technology Conference (IMTC 2007)

1-3 May 2007, Warsaw, Poland 


\title{
Error Modeling of a Fast Digital Integrator for Magnetic Measurements at CERN
}

\author{
Pasquale Arpaia ${ }^{1}, V_{\text {Vitaliano Inglese }}^{2}$, Giovanni Spiezia ${ }^{2}$, Stefano Tiso $^{3}$ \\ ${ }^{1}$ Department of Engineering, University of Sannio, Corso Garibaldi 107, 82100 Benevento, Italy. \\ Ph : +390824 305804-17, Fax: +390824 305840, E-mail: arpaia@unisannio.it \\ ${ }^{2}$ Department of Engineering, University of Naples, Federico II, Via Claudio, Napoli, Italy; \\ CERN, Dept. AT (Accelerator Technology), Group MTM, CH 1211, Genève 23, Switzerland. \\ Ph : +41 2276 76635, Fax: +41 2276 76230,E-mail: \{Vitaliano.Inglese-Giovanni.Spiezia\}@cern.ch \\ ${ }^{3}$ CERN, Dept. AT (Accelerator Technology), Group MTM, CH 1211, Genève 23, Switzerland. \\ Ph : +41 2276 76635, Fax: +41 2276 76230, E-mail: Stefano.Tiso@cern.ch
}

\begin{abstract}
A statistical behavioral modeling approach for assessing dynamic metrological performance during the concept design of accurate digitizers is proposed. A surface-response approach based on statistical experiment design is exploited in order to avoid unrealistic hypothesis of linearity, optimize simulation effort, explore systematically operating conditions, and verify identification and validation uncertainty. An actual case study on the dynamic metrological characterization of a Fast Digital Integrator for highperformance magnetic measurements at European Organization for Nuclear Research (CERN) is presented.
\end{abstract}

Keywords - Analog-to-digital converter (ADC), modeling, error analysis, statistics.

\section{INTRODUCTION}

In digital instrument design, metrological analysis by model simulation plays a key role during project assessment and enhancement [1]. During the conceptual design, several indexes of metrological performance have to be investigated in frequency and time domains (e.g., signal-to-noise-ratio, total harmonic distortion, integral and differential nonlinearity). This has to be carried out systematically, at varying operating conditions, influence parameters, and uncertainty sources, and moreover, over the input range as a whole. In particular, for highperformance accurate instruments, the effects on performance of main uncertainty and deterministic error sources have to be investigated accurately but in a generic way, i.e. independently of the architecture, defined only at the end of the conceptual design.

Modeling can be approached at several levels, according to the detail of the instrument description, useful in the different phases of the design [1]. In particular, during the conceptual design, behavioral modeling is useful for the sake of the generalization: the instrument is characterized by input-output analytical or numeral relations, without going in deep into the internal structure. In this way, a general approach can be achieved, useful in many applications, owing to its independence on the architecture of the instrument and on its physical realization.

Once the model is defined, simulations are carried out usually by following an intuitive approach: the model parameter's space is investigated by an One-Factor-AtTime strategy (OFAT) [2], [3] or by a casual variation. This can lead to misleading results, because (i) only a subspace of working conditions under investigation is explored and, (ii) moreover, often systems are not linear and parameter interactions is not revealed by OFAT analysis. Another approach is represented by Monte-Carlo simulation, capable of providing a complete exploration: the instrument model is set in each possible configuration in order to have a total comprehension of the performance landscape. However, for high-performance accurate instruments, this technique turns out to be burdensome from a computational point of view: in fact, the number of simulations to be run depends both on the number of parameters and on the width of their working range: thus such an approach can be faced only for instrument modeled by a limited number of parameters and with narrow variations of the working ranges.

In this paper, a behavioral simulation strategy based on a surface-response statistical approach is proposed. In Section II, the proposed model is illustrated, and in Section III a case study of a Fast Digital Integrator [4] for accurate dynamic magnetic measurement at European Organization for Nuclear Research (CERN) is shown.

\section{THE PROPOSAL}

In the following, (i) the basic ideas, (ii) the digitizer model, and (ii) the procedure of the proposed modeling approach are illustrated.

\section{A. Basic ideas}

The proposed approach is based on the following basic ideas:

1. comprehensive investigation of operating conditions by means of statistical experiment planning: simulations have to be carried out for each i-th metrological performance index $q_{i}$ of $\mathbf{q}^{\mathrm{T}}=\left(q_{1}, \ldots, q_{z}\right)$, which is in general a function $f_{i}$ : 


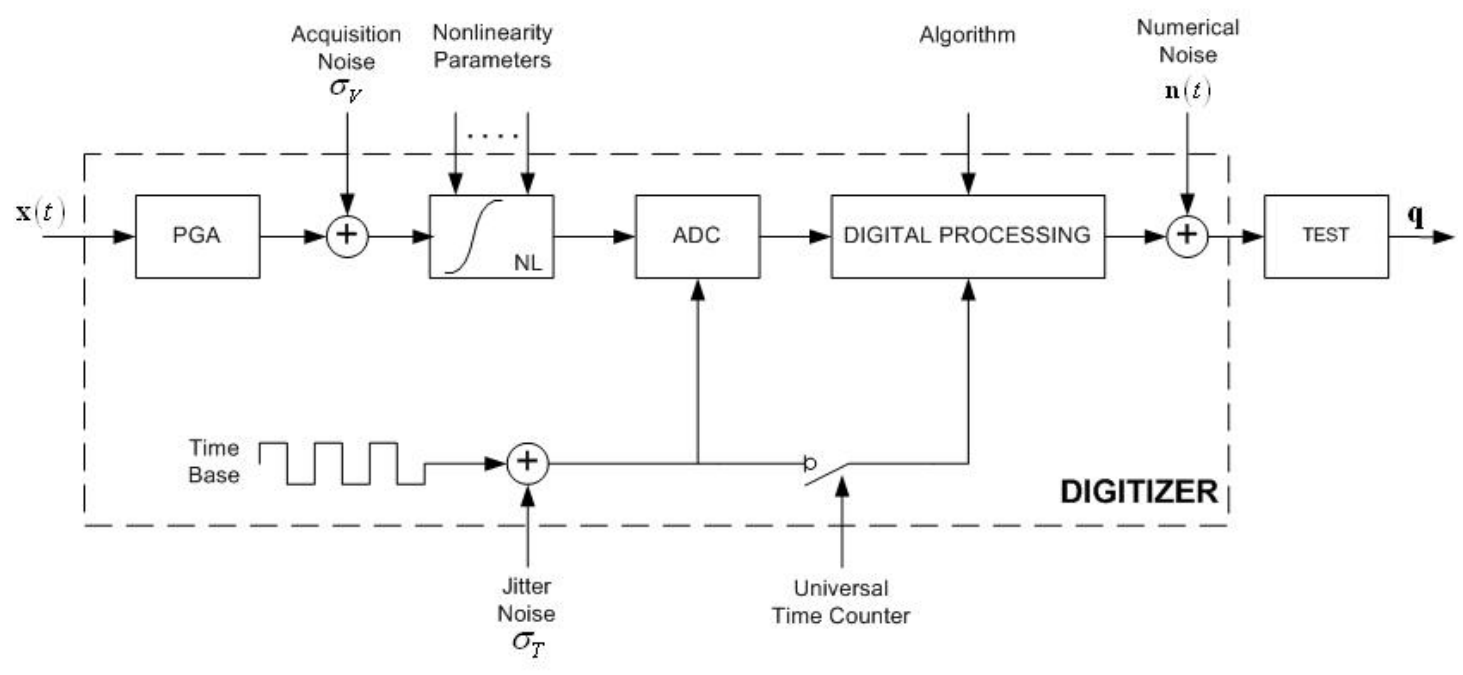

Fig. 1. Architecture of the digitizer model.

$\mathrm{q}_{\mathrm{i}}=\mathrm{f}_{\mathrm{i}}(\mathbf{x}, \mathbf{c}, \mathbf{n})$ of an input vector $\mathbf{x}^{\mathrm{T}}=\left(x_{1}, \ldots, x_{l}\right)$, the settings parameters $\mathbf{c}^{\mathrm{T}}=\left(c_{1}, \ldots, c_{m}\right)$ of the instrument, and the inner and outer uncertainty sources $\mathbf{n}^{\mathrm{T}}=\left(n_{1}, \ldots, n_{h}\right)$. The function domain $D$, $D: \mathbf{v}=\left\{v_{1} \ldots v_{p}\right\} \quad p=l+m+h, \quad$ represents the discretization of the $s$-dimensional simulation space; it is explored systematically by means of a statistical experiment planning [2], [3]: given a simulation model $f_{i}, q_{i}=f_{i}\left(v_{1}, \ldots, v_{p}\right)$, the optimum set of experiments for identifying the model, is found. The optimality is defined in terms of position inside the simulation space $D$, as well as of number of experiments for achieving a prefixed identification uncertainty.

2. behavioral architecture-independent modeling by means of a-posteriori single response-surface approach: the model $f_{i}$ is defined during the concept design of the instrument, thus it has to be generic as well as application independent. Response surfacemodeling [5], [6] allows statistical a-posteriori behavioral modeling [1]. In particular, more recent Multiple Response Surface (MRS) techniques provide better estimation of the local function nonuniformity (though biased) [7], whereas Single Response Surface (SRS) methods measure the total nonuniformity (systematic plus random). For this reasons, a SRSbased approach is more suitable to behavioral modeling.

3. nonlinear model by interaction-based representation: superposition effect-based models brings to misleading results in metrological analysis of highperformance instruments: for this reason a interactionbased model is exploited [2],[3];

4. model accuracy assessment by statistical analysis of variance: the uncertainty of the estimate is assessed by means of Multivariate ANalysis Of VAriance (MANOVA).

5. reliable model identification and validation by statistical decision making: also the model identification and validation are assessed by statistical tests; in particular, (i) for the identification, the Fisher or Pillai's trace tests [8], [9] are used for their robustness to mismatch of underlying hypotheses (especially for Fisher test), and (ii) for the validation, a $t$-test is employed in order to assess the significance of the model prediction with reference to an experiment set.

\section{B. Digitizer model}

In Fig. 1, a behavioral model of a generic digitizer for metrological purposes is shown. The input signal passes through a programmable gain amplifier (PGA), a first source of noise and distortion, modeled as an additive random noise of standard deviation $\sigma_{\gamma}$ and a nonlinear deterministic block.

Then, it is digitized by an analog-to-digital converter (ADC), introducing quantization and non-uniform sampling noise.

The time base is affected by an additive jitter noise of standard deviation $\sigma_{\tau}$.

For the sake of generalization, a Universal Time Counter (UTC) can be inserted in order to have an absolute ADCindependent time reference for accurate time-dependent measurement. In this case, the jitter noise is a relevant uncertainty source.

Finally, the last uncertainty source is the processing uncertainty modeled as an additive numerical noise $n(t)$. After a metrological test on the instrument, the performance $\boldsymbol{q}$ is measured. 


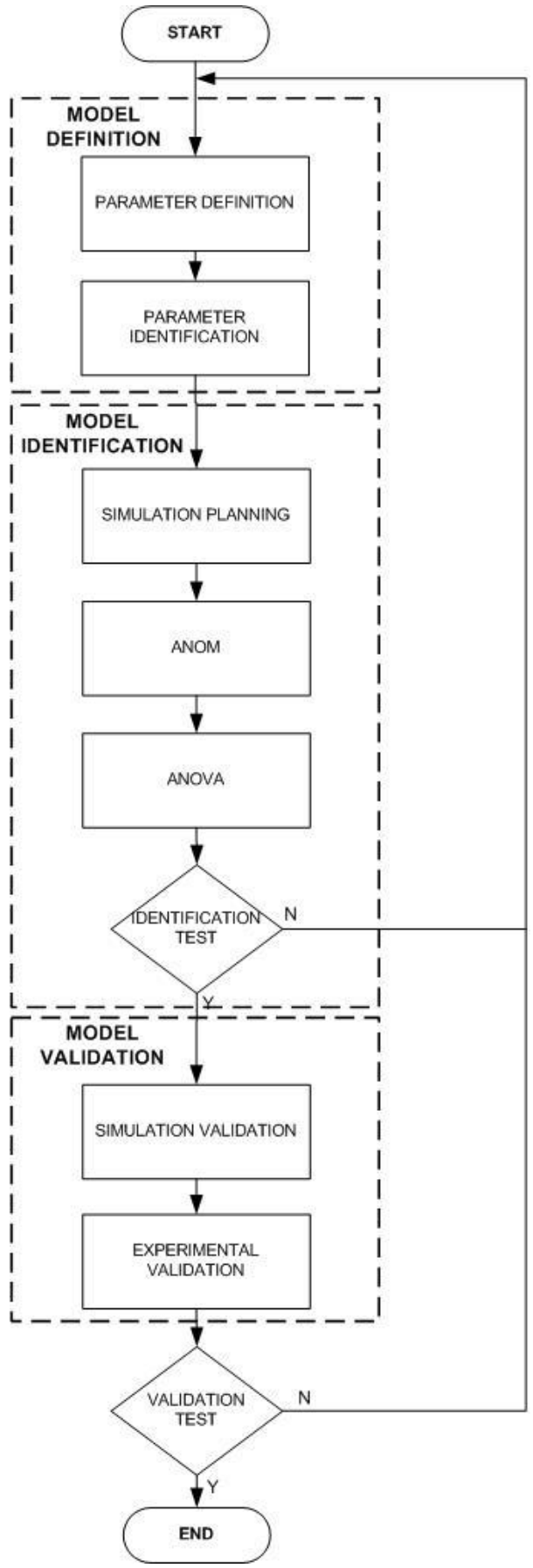

Fig. 2. Procedure for model-based analysis.

\section{Procedure}

On the basis of the above basic ideas and the related model, a procedure for analyzing the metrological performance of a digitizer is proposed through the following steps: (i) Model definition, (ii) Model Identification, and (iii) Model validation (Fig. 2).
Model Definition. The inputs for the model, $\mathbf{x}$, the parameters, $\mathbf{n}$ and $\mathbf{c}$ are defined (parameter definition) and their representation models are identified experimentally (parameter identification) (Fig. 2). In particular, by referring to the model depicted in Fig. 1, (i) the uncertainty sources and the related models $\mathbf{n}$ are represented by the standard deviations of the voltage noise of the PGA $\sigma_{\gamma}$, and the noise jitter $\sigma_{\tau}$; whereas the main deterministic error source is the nonlinearity of the acquisition chain, modeled by suitable factors; (ii) the digitizer settings, $\mathbf{c}$, are defined by the use of the UTC and by the type of the processing algorithm; and (iii) the performance index $\mathbf{q}$ as a function of $\boldsymbol{x}, \mathbf{c}$, and $\mathbf{n}$. Once the components of the vectors, $\mathbf{x}, \mathbf{c}$, and $\mathbf{n}$, are defined, they represent the continuous domain of the model function $f$. According to the particular application, the domain of the function has to be made discrete suitably, in order to carry out in a finite number of steps the simulations foreseen by the next phase.

By following the above-mentioned a-posteriori approach, at first the order of the model capable of describing suitably the system behavior is to be decided.

In the practice of experimental design, a full quadratic model is chosen in order to face the combinatorial increase of the problem dimensionality [10]. The function $\mathbf{q}=\mathbf{f}(\mathbf{x}, \mathbf{c}, \mathbf{n})=\mathbf{f}(\mathbf{v})$, describing the dependence of the performance indexes on input, setting parameters, and uncertainty sources, can expressed for the $i$-th index according to a :

$q_{i}=f_{i}(\mathbf{v})=a_{0 i}+\sum_{1 \leq k \leq p} b_{i k} v_{k}+\sum_{1 \leq k \leq j \leq p} d_{i k j} v_{k} v_{j}+\varepsilon$

where $\varepsilon$ represents the model uncertainty.

Model Identification. Afterwards, the coefficients of the response surface described by that model, which give the best fitting of simulation and experimental data have to be determined.

The suitability of the function $\mathbf{f}$ is verified by: (i) the simulation planning, (ii) the Analysis of Mean (ANOM), and (iii) the ANalysis Of VAriance (ANOVA). Then, a suitable identification test is carried out in order to validate the surface-response model (1).

$i$. Simulation Planning. The optimum subset of the simulation domain $D$ is defined by selecting an experimental plan, according to the number of parameters and their levels, as well as the desired resolution [2], i.e. the desired information about covariance between the parameters.

ii. ANOM. The analysis of mean allows the main effects of each parameter on the mean response $\mu$ to be assessed. It is estimated by the actual mean of the experiments, thus the main effect of the $k$-th parameter in any configuration $r$ can be estimated as: $\delta_{k r}=m_{k r}-\mu$; where $m_{k r}$ is the mean of $q$ in all the experiments in which the k-th parameter is in the configuration $r$ (i.e., one of the points of the discrete 
domain $D)$.

iii. $A N O V A$. The significance of the parameter effects is determined by the analysis of variance. In particular, ANOVA is aimed at determining, within a prefixed uncertainty, if a variation over the mean performance imposed by a corresponding parameter variation, is due to the parameter itself or it can be confused with the model uncertainty.

After ANOM and ANOVA analysis, an identification test is carried out in order to check the model adequacy.

In case the performance index $\mathbf{q}$ is a scalar, the Fisher test can be used to establish the global parameter F-statistic of the model [8].

In case $\mathbf{q}$ is an array, the Fisher test can be also applied on the single components of the vector. In case of MultiANOVA (MANOVA) use, the F-statistic can be determined by the Pillai's trace [9].

Model validation. A model validation can be carried out through Simulation Validation and Experimental Validation (Fig. 2).

The Simulation Validation aims to verify the goodness of the simulator in itself, as well as of the model identified. Performance is evaluated by simulations in points of the domain $D$, not considered in the simulation plan. The result is then compared with the model prediction.

The experimental validation is based on the same concept: performance of a prototype of the actual instrument in a particular functioning point are measured and then compared with the model forecast. To validate the model, the results must be consistent according to the uncertainty $\varepsilon$ of the model.

\section{EXPERIMENTAL CASE STUDY OF A FAST DIGITAL INTEGRATOR AT CERN}

The proposed method was validated through experiments on the Fast Digital Integrator (FDI) [4] in the laboratory of Magnet Test \& Measurement Group (Accelerator Technologies Dept) of CERN.

In the following, (i) the FDI conceptual design, (ii) the model definition, (iii) the model Identification, (iv) and the model validation are illustrated.

\section{A. FDI Conceptual Design}

At CERN, during the last year, a Fast Digital Integrator has been proposed for a real-time on-board integration of the transducer signal [4]. This instrument can analyze the magnetic flux over a bandwidth larger than state-of-art solutions, with a higher accuracy, owing to its 18 bitresolution and $500 \mathrm{kS} / \mathrm{s}$-rate digital conversion, as well as higher precision, owing to its self-correction of offset and gain errors.

In synthesis, the FDI has to sample an analog signal and to output the computed integral at a trigger frequency

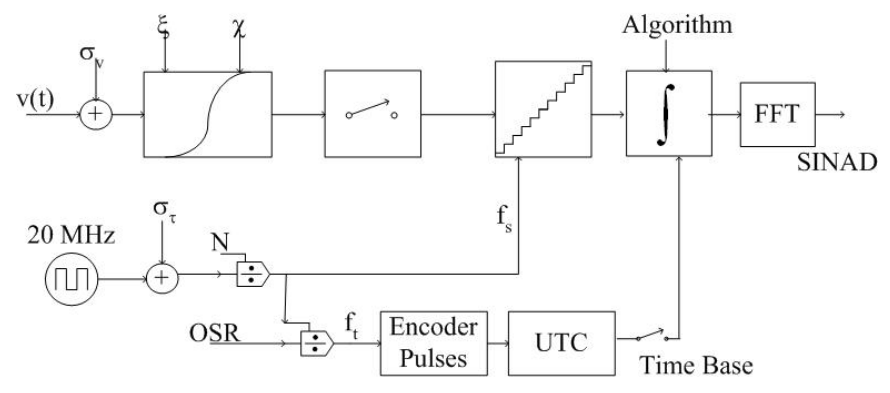

Fig. 3. FDI behavioral model

determined by an encoder mounted on the shaft of a coil rotating inside the superconducting magnet under test (test technique of rotating coil). In the FDI architecture, the input signal arising from the transducer, put into the superconducting magnet under test, is conditioned by a low-noise low-distortion custom differential PGA. Then is sampled in the time domain by an 18-bit SAR ADC. The ADC data are integrated digitally by means of a DSP, in the angular domain, by exploiting the trigger pulses of an encoder. These pulse events are measured by a time-base with a resolution of $50 \mathrm{~ns}$ to provide a fine link between the time and the angular domain. A field-programmable gate array (FPGA) acts as I/O processor: it supervises the PGA operations at low level and provides the interface for the board bus PXI.

\section{B. The Model Definition}

In the FDI conceptual project, the design team is interested in investigating the impact of possible nonideality sources (n) on the dynamic distortion (q) of the output, at varying the working operating conditions, defined by the measurand ( $\mathbf{x})$ and by the design settings (c).

As a first step, a first-order simple model is exploited to describe the dependency of $\mathbf{x}, \mathbf{c}, \mathbf{n}$ on $\mathbf{q}$ (Eq. 1 with $\mathrm{d}_{\mathrm{ikj}}=$ $0)$. If it would turn out to be inadequate, a second-order, more accurate model, including also interactions among parameters, will be considered.

The input $\mathbf{x}$ of the FDI model (Fig. 3) is the OverSampling Ratio (OSR), defined as the ratio between the ADC sampling rate $f_{s}$ and the trigger frequency $f_{t}$. The model output $\mathbf{q}$ is the SIgnal-to-Noise And Distortion Ratio (SINAD) of the computed integral. Such a parameter is evaluated on the flux increment computed between two trigger pulses.

The control parameters c are the design settings, namely the type of integration algorithm (rectangular or trapezoidal), and the possibility of using the UTC for a fine link between angular and time domains (Fig. 3).

The influence parameters $\mathbf{n}$ are the time stamp jitter noise $\left(\sigma_{\tau}\right)$, the acquisition noise $\left(\sigma_{\mathrm{v}}\right)$, and the parameters for representing $\mathrm{ADC}$ nonlinearity $(\chi, \xi)$ [11]. In particular, the parameter $\chi$ takes into account the asymmetry of the transfer function, while the parameter $\xi$ the exponential non linearity.

By summarizing, the FDI model arrays are: 
Table 1. Numerical values of the parameter model

\begin{tabular}{|c|c|c|c|}
\hline & \multicolumn{3}{|c|}{ Levels } \\
\hline & $\mathbf{1}$ & $\mathbf{2}$ & $\mathbf{3}$ \\
\hline Algorithm & rectangular & trapezoidal & $\#$ \\
\hline $\begin{array}{c}\text { Universal Time } \\
\text { Counter }\end{array}$ & present & absent & $\#$ \\
\hline $\begin{array}{c}\text { Time Stamp Jitter } \\
\text { Acquisition Noise }\end{array}$ & $5 \cdot 10^{-15}$ & $5 \cdot 10^{-13}$ & $5 \cdot 10^{-12}$ \\
\hline$\chi$ & $5.0 \cdot 10^{-4}$ & $3.7 \cdot 10^{-4}$ & $2.7 \cdot 10^{-4}$ \\
\hline$\xi$ & $500 \cdot 10^{-6}$ & $1.6 \cdot 10^{-4}$ & $5.0 \cdot 10^{-5}$ \\
\hline $\boldsymbol{z}$ & $250 \cdot 10^{-6}$ & $1 \cdot 10^{-6}$ \\
\hline
\end{tabular}

$x=O S R ; \quad q=S I N A D ; \quad \mathbf{c}^{T}=($ Algorithm,$U T C) ;$

$\mathbf{n}^{T}=\left(\sigma_{T}, \sigma_{V}, \chi, \xi\right)$.

The working range of each parameter and its levels are determined according the following procedures:

OSR: by assuming an ADC sampling rate of $125 \mathrm{kS} / \mathrm{s}$, three different values of OSR were chosen, $(1,12.5125)$ in order to evaluate the performance for different values of trigger frequency $\left(\mathrm{f}_{\mathrm{t}}\right), 125 \mathrm{kHz}, 10 \mathrm{kHz}$, and $1 \mathrm{kHz}$.

Time stamp jitter: it was defined through the reference clock data sheet.

Acquisition noise: by assuming a normal distribution, the acquisition noise was derived experimentally, according to:

$$
\begin{aligned}
& S N R=10 \cdot \log \frac{P_{\text {sig }}}{P_{\text {noise }}}=10 \cdot \log \frac{P_{\text {sig }}}{\sigma_{V}^{2}} ; \\
& \sigma_{V}^{2}=\frac{P_{\text {sig }}}{10^{\left(\frac{S N)}{10}\right)}} ;
\end{aligned}
$$

where SNR is the Signal-to-Noise Ratio in $\mathrm{dB}, \mathrm{P}_{\text {sig }}$ is the input signal power, and $\mathrm{P}_{\text {noise }}$ is the noise power over all the bandwidth, expressed by the variance $\sigma^{2}$ v.

$A D C$ nonlinearity parameters: according to a twoparameters ADC model [11], a direct relation between the second and third harmonics amplitude and $\xi$ and $\chi$ is derived from the FFT of the output:

$$
\begin{aligned}
& \chi \simeq 2 \frac{A m p_{I I}}{\left(A m p_{I}\right)^{2}} ; \\
& \xi \simeq 4 \frac{A m p_{I I I}}{A m p_{I}}
\end{aligned}
$$

where $A m p_{i}$ represents the amplitude of the $i$-th harmonic. In Tab.1, the numerical values of the FDI parameter model, selected such as above mentioned, are reported.

\section{Model Identification}

According to the procedure of Fig. 2, the model is identified by: (i) Simulation Planning, (ii) ANOM and ANOVA, and (iii) Fischer test.

Simulation Planning: A Resolution III standard Taguchi plan L18 is used, owing its capability of exploring a
Table 2. ANOM at $1 \mathrm{kHz}$ trigger frequency.

\begin{tabular}{|c|c|c|c|c|}
\hline & \multicolumn{3}{|c|}{ Levels } & \\
\hline & $\mathbf{1}$ & $\mathbf{2}$ & $\mathbf{3}$ & $\boldsymbol{\Delta}$ \\
\hline Algorithm & -0.01 & 0.01 & & 0.01 \\
\hline Time Stamp Jitter & 0.32 & -0.16 & -0.16 & 0.49 \\
\hline Acquisition Noise & 0.31 & -0.15 & -0.16 & 0.47 \\
\hline$\chi$ & -9.50 & 0.25 & 9.25 & 18.75 \\
\hline$\xi$ & -0.78 & 0.31 & 0.46 & 1.24 \\
\hline
\end{tabular}

combinatorial space generated by up to seven 3-level parameters and one 2-level parameter, according to a firstorder model.

A first simulation run showed that the UTC has a largely predominating influence on the performance, by making the other parameters negligible. Thus, the UTC was permanently used in the further simulation planning and the only parameter control left is the algorithm. In this case, one 2-level (integration algorithm type) and four 3level (time stamp jitter, acquisition noise, $\xi$, and, $\chi$ ) parameters are considered, thus 8 degrees of freedom are left for determining the model uncertainty.

ANOM and ANOVA. In Tab. 2, the effects of each parameter on the average SINAD are shown, for an OSR of 125 due to an ADC sampling rate of $125 \mathrm{kS} / \mathrm{s}$ and a trigger frequency equal to $1 \mathrm{kHz}$. By the table, an effectiveness of the non-linearity parameter $\xi$ and $\zeta$ seems to rise up. Furthermore, the time stamp jitter and the acquisition noise have the largest influence on the SNR. Tab. 3 reports about the ANOVA results, by showing (i) the contribution of each parameter to the performance variance $\left(\sigma^{2}\right)$, (ii) the corresponding F-statistic $(\mathrm{F})$, and (iii) the $\mathrm{P}$-parameter $(\mathrm{P})$ pointing out the probability that a parameter does not affect $\mathbf{q}$ with a confidence level of $1 \%$. The SINAD is a scalar quantity, thus the goodness of the first-order model was verified by a Fisher test. As larger the index $F_{i}$ is, as the variation over the mean is due to the parameter variation and not to the model uncertainty.

Thus, the F-statistic and the P-parameter of Tab. 3 show that the most significant parameter is the non-linearity

Table 3. ANOVA at $1 \mathrm{kHz}$ trigger frequency.

\begin{tabular}{c|cccc} 
& $\gamma$ & $\sigma^{2}$ & $\mathbf{F}_{\mathbf{i}}$ & $\mathbf{P}$ \\
\hline Model & 5 & 192.9 & 22.0 & $<1 \cdot 10^{-4}$ \\
\hline Algorithm & 1 & $2.7 \cdot 10^{-3}$ & $3.1 \cdot 10^{-4}$ & $9.9 \cdot 10^{-1}$ \\
Time Stamp Jitter & 1 & $6.2 \cdot 10^{-1}$ & $7.2 \cdot 10^{-2}$ & $7.9 \cdot 10^{-1}$ \\
Acquisition Noise & 1 & $5.9 \cdot 10^{-1}$ & $6.9 \cdot 10^{-2}$ & $8.0 \cdot 10^{-1}$ \\
$\chi$ & 1 & 962.5 & 111.0 & $<1 \cdot 10^{-4}$ \\
$\xi$ & 1 & 2.1 & $2.4 \cdot 10^{-1}$ & $6.3 \cdot 10^{-1}$ \\
Error & 12 & 8.6 & &
\end{tabular}




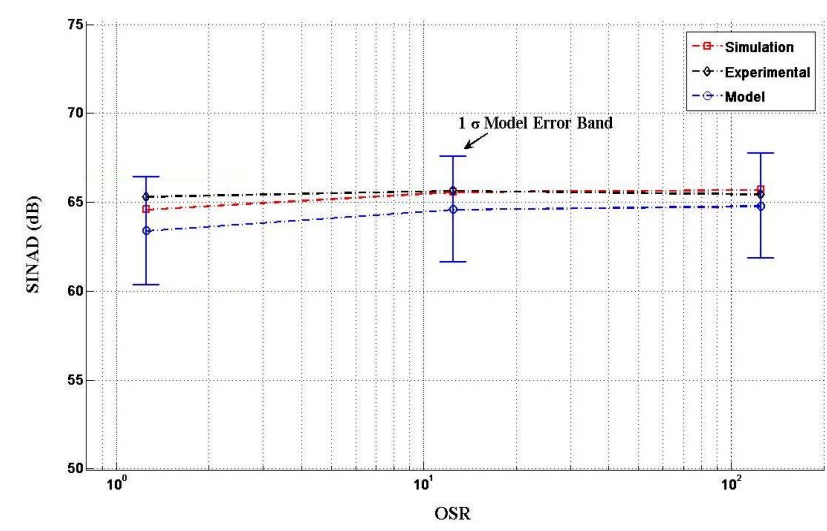

Fig. 4. Comparison among the simulated performance, experimental performance and model predictions.

parameter $\chi$. As a matter of fact, it is directly related to the harmonics of the signal to be integrated. Actually, its effect cannot be avoided, but the proposed technique allowed the reachable improvement to be pointed out, if the FDI analog part is optimized, or if a suitable correction is carried out.

The Fisher test confirms the suitability of the first-order model to represent the FDI behavior, thus, the weight coefficients of the model parameter were determined:

$$
q=C+k \tilde{\chi}=69.1+8.61 \cdot \tilde{\chi},
$$

where

$$
\begin{aligned}
& \tilde{\chi}=\frac{\log (\chi)-\frac{\hat{\chi}_{\max }-\hat{\chi}_{\min }}{2}}{\hat{\chi}_{\text {max }}} \\
& \hat{\chi}_{\text {max }}=\log \left(\chi_{\text {max }}\right) \text { and } \hat{\chi}_{\text {min }}=\log \left(\chi_{\text {min }}\right)
\end{aligned}
$$

and $\chi_{\max }$ and $\chi_{\min }$ are, respectively the maximum and minum value of the parameter $\chi$ (Tab. 1$)$.

Eq. 6 expresses the dependence of the SINAD index on the non-linearity parameter $\chi$, related to the harmonic distortion.

\section{Model Validation}

In Fig. 4, the FDI performance in terms of SINAD is depicted for different value of the OSR. The graph shows that the predictions of the model are consistent with the simulated results, as well as with the experimental results within the model uncertainty.

The model is highly helpful in determining the sensitivity of the FDI overall distortion to the ADC nonlinearity. Moreover, the conclusions of model analysis point out that the most important distortion to be corrected is the asymmetry of the transfer function represented by $\chi$. This is a valuable requirement for the correction procedure of the dynamic distortion to be realized.

\section{CONCLUSIONS}

In this paper, an error modeling technique of accurate digitizers for metrological purposes is presented. A systematic procedure allows the behavioral model of the device to be defined, identified, and validated.

As a case study, a Fast Digital Integrator, developed at CERN for magnetic measurements, was analyzed and modeled to understand its main uncertainty sources. The results show the practical usefulness of the proposed approach in defining the impact of uncertainty source on working conditions and design choices during the concept design of a digital instrument. As a matter of fact, the model pointed out that the most important distortion to be corrected is the asymmetry of the transfer function. This is an important indication for the FDI correction procedure to be realized.

\section{ACKNOWLEDGEMENTS}

This work was sponsored by CERN trough the agreement No K 1201/AT/LHC with the Department of Engineering, University of Sannio, whose support authors gratefully acknowledge. Authors thank Luca Bottura, Felice Cennamo, Domenico Della Ratta, for their useful cooperation.

\section{REFERENCES}

[1] P. Arpaia, P.Daponte, S.Rapuano, "A state of the art on ADC Modelling", Computer Standards and Interfaces, vol. 26, , pp.31-42, 2003.

[2] G. E. P. Box, W. G. Hunter and J. Stuart Hunter, J. Wiley, "Statistics for Experimenters: Design, Innovation, and Discovery" (Second Edition), New York, 2005.

[3] G. E. P. Box, N. R. Draper, J. Wiley, "Empirical Model-Building and Response Surfaces", New York, 1987.

[4] P. Arpaia, A. Masi, G. Spiezia, "A Digital Integrator for Fast and Accurate Measurement of Magnetic Flux by Rotating Coils", IEEE Transactions on Instrumentation \& Measurements, 2007, in press.

[5] Douglas C. Montgomery, Raymond H. Myers, "Response Surface Methodology: Process and Product Optimization Using Designed Experiments", Wiley-Interscience, Jan. 1995.

[6] M. Papila, R. T. Haftka, "Response Surface Approximations: Noise, Error Repair, and Modeling Errors," AIAA Journal, Vol. 38, N. 12, Dec. 2000.

[7] T. H. Smith, B. E. Goodlin, D. S. Boning, H. H. Sawin, "A Statistical Analysis of Single and Multiple Response Surface Modeling”, IEEE Transactions on semiconductor manufcturing, Vol. 12, N. 4, pp. 419430, Nov. 1999.

[8] D. E. Johnson, "Applied multivariate methods for data analysis". Pacific Grove, CA, Duxbury Press.

[9] B. G. Tabachnick, L. S. Fidell, "Using Multivariate Statistics", 4th Ed. New York, Allyn \& Bacon.

[10] Bellman, R., Dynamic Programming, Princeton University Press, Princeton, New Jersey, 1957.

[11] K. Kim, "Analog-to-Digital Conversion and Harmonic Noises Due to the Integral Nonlinearity", IEEE Transactions on Instrumentation and Measurement, vol. 43, No. 2, April 1994. 